\title{
Safety profile of bivalirudin in Chinese female patients undergoing percutaneous coronary intervention: a multi-center study
}

Fan $\mathrm{Wu}^{1 \dagger}$, Xueying Liư ${ }^{2 \dagger}$, Huazhong Ran ${ }^{3}$, Qiwei Tang ${ }^{4}$, Cheng Zhong ${ }^{5}$, Yanqing Wu ${ }^{6 *}$ and Jun Xiao ${ }^{7 *}$

\begin{abstract}
Background: The present study aimed to comprehensively investigate the occurrence and risk factors of adverse events (AEs) or adverse drug reactions (ADRs) (especially for thrombocytopenia and bleeding) in Chinese female patients receiving bivalirudin during percutaneous coronary intervention ( $\mathrm{PCl}$ ).
\end{abstract}

Methods: A total of 918 female patients from 27 Chinese medical centers took bivalirudin as anticoagulant for PCI were enrolled in this prospective, multi-center, intensive monitoring study. Safety data (AEs, ADRs, thrombocytopenia and bleeding) were collected from admission to $72 \mathrm{~h}$ post bivalirudin administration; then, patients were followed up at the $30^{\text {th }}$ day with the safety data collected as well.

Results: One hundred and twenty (13.1\%) patients occurred AEs, among which 7 (0.8\%) cases experienced severe AEs, and $2(0.2 \%)$ cases died. Besides, 40 (4.4\%) patients occurred bivalirudin-related ADRs, in which $3(0.3 \%)$ cases experienced severe ADRs, but $0(0.0 \%)$ cases died. It was of note that $27(2.9 \%)$ and $13(1.4 \%)$ patients experienced thrombocytopenia and bleeding, respectively. Subsequent multivariate analyses observed that: clinical presentation of spontaneous coronary artery dissection (SCAD) (odds ratio $(O R)=3.191, P=0.004), C R U S A D E$ high risk $(O R=2.075$, $P=0.031)$, multiple culprit vessel $(O R=2.328, P=0.019)$ independently correlated with higher risk of bivalirudinrelated ADRs; clinical presentation of SCAD $(\mathrm{OR}=4.388, P=0.002)$ and multiple culprit vessel $(\mathrm{OR}=2.974, P=0.010)$ independently linked with raised thrombocytopenia risk; history of diabetes mellitus $(O R=5.227, P=0.007)$ and CRUSADE high risk ( $O R=4.475, P=0.016$ ) were independent factor related to elevated bleeding risk.

Conclusion: Bivalirudin is well tolerated with low ADRs, thrombocytopenia and bleeding incidences in Chinese female patients undergoing PCl.

Keywords: Bivalirudin, Percutaneous coronary intervention, Female, Adverse events and adverse drug reactions, Thrombocytopenia and bleeding

*Correspondence: quyanjie490429890@163.com; qiaoji3208262@163.com

${ }^{\dagger}$ Fan Wu and Xueying Liu contributed equally to this work.

${ }^{6}$ Department of Cardiovascular Medicine, The Second Affiliated Hospital of Nanchang University, 1 Minde Road, Donghu District, Nanchang 330006, China

${ }^{7}$ Department of Cardiovascular Medicine, Chongqing University Central Hospital, No. 1 Jiankang Road, Yuzhong District, Chongqing 400014, China

Full list of author information is available at the end of the article

\section{Background}

Since the introduction of percutaneous coronary intervention (PCI) with or without drug-eluting stents (DES), it has been widely used to treat coronary artery disease (CAD) with good efficacy and tolerant adverse reactions $[1,2]$. Gender differences in CAD commonly exist in several aspects, such as coronary anatomy, risk factors, comorbidities, CAD pathophysiology, clinical presentation response to pharmacotherapy mainly due to sex 
hormone variations [3-5]; meanwhile, gender also affects outcomes in CAD patients after PCI $[5,6]$. Therefore, it is necessary to dig more information about the PCI application in female CAD patients.

Bivalirudin, as a synthetic congener of the naturally occurring drug hirudin, conquers several shortcomings of traditional indirect thrombin inhibitor such as heparin [7-9]. As for clinical utility, several large-scale, randomized, controlled trials have demonstrated the superiority of bivalirudin over heparin with or without Glycoprotein (GP) IIb/IIIa inhibitor in CAD patients underwent PCI [9-14]. However, there are few reports in terms of the adverse events (AEs) or adverse drug reactions (ADRs) (especially thrombocytopenia and bleeding) of bivalirudin as anticoagulant during $\mathrm{PCI}$ in the specific female patients, not to mention the lack of data on bivalirudin in Chinese patients.

Therefore, the current prospective, multi-center, intensive monitoring study aimed to comprehensively investigate the occurrence and risk factors of AEs and ADRs (especially for thrombocytopenia and bleeding) in Chinese female patients receiving bivalirudin as an anticoagulant during $\mathrm{PCI}$.

\section{Methods}

\section{Patients}

A total of 918 female patients' data were abstracted from a prospective, multi-center, intensive monitoring study which enrolled 3049 patients who underwent PCI and received bivalirudin as anticoagulant in 27 Chinese medical centers, between July 2018 and June 2019, aiming to further evaluate the safety of bivalirudin in a wide range of population. These 918 patients were chosen based on the criterium of being females.

In detail, the inclusion criteria were: (1) underwent PCI or percutaneous coronary angioplasty (PTCA); (2) used bivalirudin as anticoagulant; (3) age over 18 years; (4) female patients; (5) understood the study content and voluntarily participated in the study. Patients without use of bivalirudin were excluded from the study.

The study was conducted in accordance with the Declaration of Helsinki, and was approved by the Ethics Committee of the Chongqing University Central Hospital and all patients provided the written informed consents.

\section{Collection of clinical data}

The following clinical data were collected: (i) demographic characteristics; (ii) medial history; (iii) clinical presentation: unstable angina (UA); ST-segment elevation myocardial infarction (STEMI); non-STsegment elevation myocardial infarction (NSTMI) and spontaneous coronary artery dissection (SCAD); (iv) CRUSADE score (Can Rapid Risk Stratification of
Unstable Angina Patients Suppress Adverse Outcomes with Early Implementation of the ACC/AHA Guidelinesbleeding score[15]); (v) PCI characteristics; (vi) administration of bivalirudin (vii) combined with GP IIb/IIIa inhibitors; (viii) thrombolysis in myocardial infarction (TIMI) flow grade (pre-procedure) and TIMI flow grade (post-procedure).

\section{Collection of safety data}

Safety data were collected from hospital admission to $72 \mathrm{~h}$ after completion of bivalirudin administration. In addition, patients were followed up at the $30^{\text {th }}$ day "in person", and the data were also collected at that time. ADRs were classified using the Systematic Organ Classification (SOC) and Preferred Term (PT) from the International Conference on the Coordination of International Drug Registration (ICH) Medical Dictionary for Regulatory Activities (MedDRA) 23.0.

\section{Definitions}

AEs were defined as any unfavorable and unintended sign (including an abnormal laboratory finding), symptom, or disease that is temporarily associated with the use of a medical treatment that may or may not be considered related to the medical treatment. ADRs were defined as the harmful reactions of qualified drugs which was irrelevant to the purpose of medication under normal usage and dosage. Severe adverse events (SAEs) and severe adverse drug reactions (SADRs) were defined as one of the following events: (i) resulting in death; (ii) life-threatening consequences; (iii) leading to carcinogenesis, teratogenesis and birth defects; (iv) resulting in significant or permanent human disability or organ function damage; (v) resulting in hospitalization or prolonged length of stay; (vi) leading to other important medical events, and if not treated, the above listed conditions may occur. The severity of AEs and ADRs was classified according to the following criteria: (i) mild: symptoms were transient and did not affect the patient's normal daily activities; (ii) moderate: symptoms were significant and affect the patient's normal daily activities, but tolerable, which were not required discontinuation of medication; (iii) severe: symptoms were obvious, intolerable and affected the patient's normal daily activities, which were required discontinuation of medication. The bleeding was defined and graded in terms of Bleeding Academic Research Consortium (BARC) consensus classification criteria [16]. The thrombocytopenia was defined as blood platelet below $75 \times 10^{9} / \mathrm{L}$.

\section{Statistical analysis}

SAS 9.4 (SAS Institute, Inc., Cary, North Carolina, USA) was applied to complete data analysis. Normally 
distributed continuous variable was presented as mean value \pm standard deviation, and categorized variable was expressed as count (percentage). Summaries of all AEs were calculated based on cases. If a case suffered from the same $\mathrm{AE}$ repeatedly, the most severe $\mathrm{AE}$ was reported in the study. Univariate logistic regression analysis was carried out to assess the factors related to risk of ADRs, thrombocytopenia and bleeding events; then the covariates with $P$ value less than 0.05 in the univariate logistic regression analysis were further selected to be included in multivariable logistic model analysis (method: enter, in the SPSS software). $P$ value $<0.05$ was considered statistically significant.

\section{Results}

\section{Study flow}

Three thousand and forty-nine patients who underwent $\mathrm{PCI}$ and received bivalirudin as anticoagulant in $27 \mathrm{Chi}-$ nese medical centers were initially enrolled, then 918 female patients were sorted out for the analysis in this current study (Fig. 1). Safety data collection was performed within 72 -h close monitor and at $30^{\text {th }}$ day follow up. AEs, ADRs, thrombocytopenia and bleeding information, as well as their risk factors were evaluated.

\section{Patients' characteristics}

A total of 918 female patients receiving bivalirudin as an anticoagulant during PCI were enrolled with an age of $68.8 \pm 9.2$ years (Table 1). 360 (39.2\%), 329 (35.8\%), $129(14.1 \%), 99(10.8 \%)$ patients presented with unstable angina (UA), STEMI, NSTMI, and SCAD, respectively. Other detailed patients' characteristics and PCI characteristics were exhibited in Table 1.

\section{AEs, ADRs, thrombocytopenia and bleeding}

One hundred and twenty (13.1\%) patients occurred AEs, among which $7(0.8 \%)$ cases experienced SAEs, and 2 (0.2\%) cases died. In addition, 40 (4.4\%) patients occurred bivalirudin-related ADRs, in which $3(0.3 \%)$ cases experienced SADRs, but $0(0.0 \%)$ cases died (Table 2$)$. The detailed classifications of AEs and bivalirudin-related ADRs in SOC were presented in Table 3, which observed that gastrointestinal disorders and blood and lymphatic system disorders were the most common AEs and bivalirudin-related ADRs. In addition, it was noteworthy that 27 (2.9\%) and 13 (1.4\%) patients experienced thrombocytopenia and bleeding, respectively (Table 2).

\section{Factors related to bivalirudin-related ADRs risk}

Univariate analyses showed that clinical presentation of UA was correlated with lower risk of bivalirudin-related ADRs $(P=0.006)$, whereas clinical presentation of SCAD $(P=0.001)$, CRUSADE high risk $(P=0.005)$, multiple culprit vessel $(P=0.048)$, preoperative or intraoperative administration of bivalirudin $(P=0.026)$ were associated with higher risk of bivalirudin-related ADRs. Subsequent multivariate analyses revealed that clinical manifestations of SCAD $(P=0.004)$, CRUSADE high risk $(P=0.031)$, multiple culprit vessel $(P=0.019)$ independently correlated with higher risk of bivalirudin-related ADRs (Table 4).

\section{Factors related to thrombocytopenia and bleeding risk}

Univariate analyses observed that clinical presentation of UA was associated with reduced thrombocytopenia risk $(P=0.032)$, whereas clinical presentation of SCAD $(P<0.001)$, multiple culprit vessel $(P=0.022)$ and

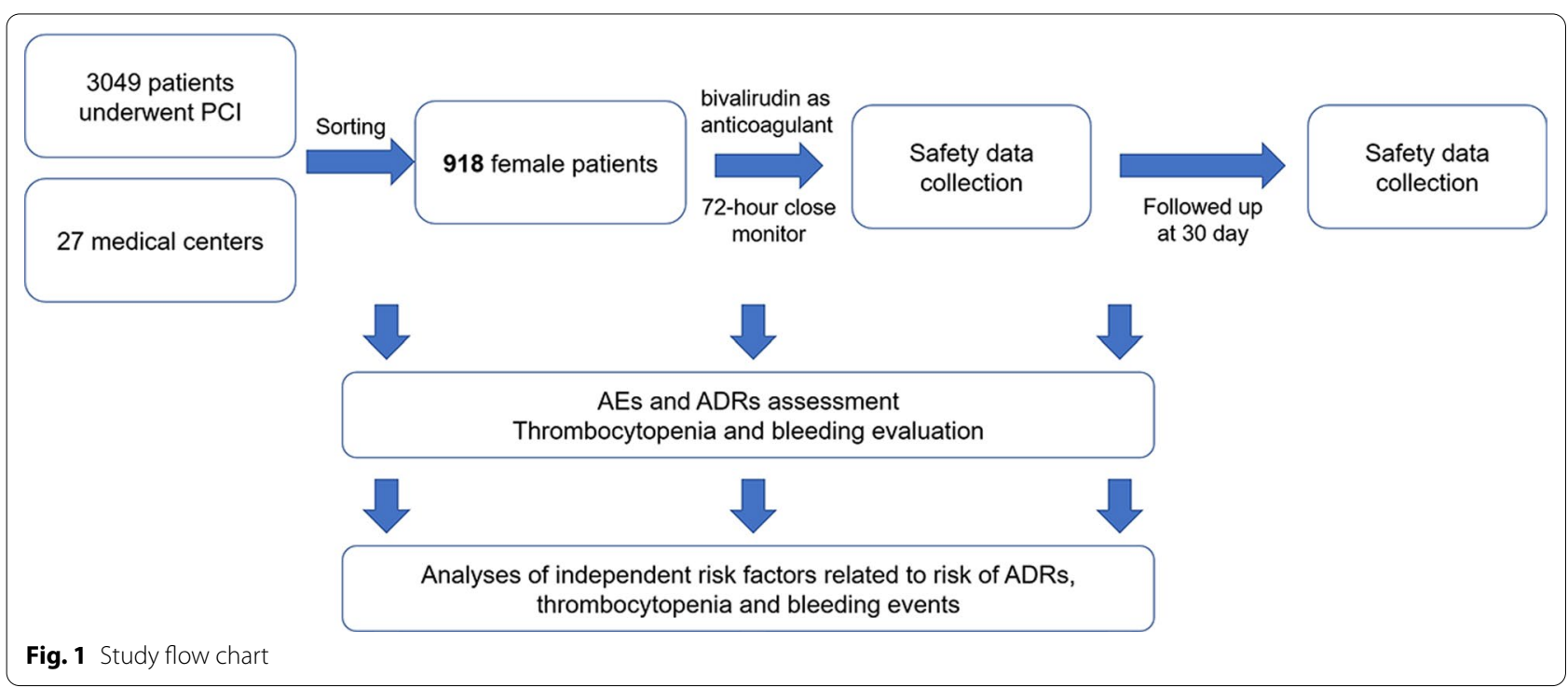


Table 1 Clinical characteristics of female patients

\begin{tabular}{|c|c|}
\hline Items & Patients ( $\mathrm{N}$ \\
\hline \multicolumn{2}{|l|}{ Demographic characteristics } \\
\hline Age (years), mean $\pm S D$ & $68.8 \pm 9.2$ \\
\hline $\mathrm{BMI}\left(\mathrm{kg} / \mathrm{m}^{2}\right)$, mean $\pm \mathrm{SD}$ & $24.5 \pm 25.5$ \\
\hline \multicolumn{2}{|l|}{ Medical history } \\
\hline History of diabetes mellitus, No. (\%) & $275(30.0)$ \\
\hline History of allergy, No. (\%) & $105(11.4)$ \\
\hline History of cardiac surgery, No. (\%) & $72(7.8)$ \\
\hline History of renal function impairment, No. (\%) & $27(2.9)$ \\
\hline History of critical respiratory disease, No. (\%) & $20(2.2)$ \\
\hline \multicolumn{2}{|l|}{ Clinical presentation } \\
\hline UA, No. (\%) & $360(39.2)$ \\
\hline STEMI, No. (\%) & $329(35.8)$ \\
\hline NSTMI, No. (\%) & $129(14.1)$ \\
\hline SCAD, No. (\%) & $99(10.8)$ \\
\hline Others, No. (\%) & $1(0.1)$ \\
\hline \multicolumn{2}{|l|}{ CRUSADE score } \\
\hline Mean \pm SD & $35.4 \pm 12.7$ \\
\hline \multicolumn{2}{|l|}{ Risk stratification, No. (\%) } \\
\hline Very low risk $(\leq 20)$ & $81(8.8)$ \\
\hline Low risk $(21-30)$ & $275(30.0)$ \\
\hline Moderate risk (31- 40) & $278(30.3)$ \\
\hline High risk (41 - 50) & $158(17.2)$ \\
\hline Very high risk (> 50) & $111(12.1)$ \\
\hline Unknown & $15(1.6)$ \\
\hline \multicolumn{2}{|l|}{$\mathrm{PCl}$ characteristics } \\
\hline \multicolumn{2}{|l|}{ Operative timing, №. (\%) } \\
\hline Emergency operation & $349(38.0)$ \\
\hline Elective operation & $569(62.0)$ \\
\hline \multicolumn{2}{|l|}{ Types of coronary interventional therapy, №. (\%) } \\
\hline Stent implantation & $872(95.0)$ \\
\hline Balloon dilatation & $37(4.0)$ \\
\hline Thrombus aspiration & $0(0.0)$ \\
\hline Others & $9(1.0)$ \\
\hline \multicolumn{2}{|l|}{ Types of stents, No. (\%) } \\
\hline Drug stent & $860(93.7)$ \\
\hline Bare stent & $15(1.6)$ \\
\hline Unknown & $43(4.7)$ \\
\hline \multicolumn{2}{|l|}{ Arterial access, №. (\%) } \\
\hline Brachial artery & $1(0.1)$ \\
\hline Femoral artery & $66(7.2)$ \\
\hline Radial artery & $848(92.4)$ \\
\hline Others & $3(0.3)$ \\
\hline \multicolumn{2}{|l|}{ Culprit vessel, No. (\%) } \\
\hline Single & $715(77.9)$ \\
\hline Multiple & $203(22.1)$ \\
\hline \multicolumn{2}{|l|}{ Administration of bivalirudin } \\
\hline Preoperative or intraoperative, №. (\%) & $31(3.4)$ \\
\hline Postoperative $\leq 4$ h, No. (\%) & $779(84.9)$ \\
\hline Postoperative > 4 h, №. (\%) & $108(11.7)$ \\
\hline
\end{tabular}

Table 1 (continued)

\begin{tabular}{ll}
\hline Items & Patients (N=918) \\
\hline \multicolumn{1}{c}{ Combined with GP IIb/IIla inhibitors, No. (\%) } & $663(72.2)$ \\
\hline TIMI flow grade (pre-procedure) & \\
0, No. (\%) & $241(26.3)$ \\
1, No. (\%) & $145(15.8)$ \\
2, No. (\%) & $86(9.4)$ \\
3, No. (\%) & $442(48.1)$ \\
Unknown, No. (\%) & $4(0.4)$ \\
TIMI flow grade (post-procedure) & \\
0, No. (\%) & $4(0.4)$ \\
1, No. (\%) & $4(0.4)$ \\
2, No. (\%) & $14(1.5)$ \\
3, No. (\%) & $895(97.6)$ \\
Unknown, No. (\%) & $1(0.1)$ \\
\hline
\end{tabular}

SD, standard deviation; BMI, body mass indexes; $\mathrm{UA}$, unstable angina; STEMI, ST-segment elevation myocardial infarction; NSTMI, non-ST-segment elevation myocardial infarction; SCAD, spontaneous coronary artery dissection; CRUSADE, Can Rapid Risk Stratification of Unstable Angina Patients Suppress Adverse Outcomes with Early Implementation of the ACC/AHA Guidelines; PCI percutaneous coronary intervention; GP, glycoprotein; TIMI, thrombolysis in myocardial infarction

Table 2 Summary of AEs and bivalirudin-related ADRs

\begin{tabular}{lc}
\hline Items & Incidence, No. (\%) \\
\hline Total AEs & $120(13.1)$ \\
SAEs & $7(0.8)$ \\
Hospitalization & $4(0.4)$ \\
Death & $2(0.2)$ \\
Other important medical events & $1(0.1)$ \\
Total bivalirudin-related ADRs & $40(4.4)$ \\
SADRs & $3(0.3)$ \\
Hospitalization & $1(0.1)$ \\
Death & $0(0.0)$ \\
Other important medical events & $2(0.2)$ \\
Thrombocytopenia & $27(2.9)$ \\
Bleeding & $13(1.4)$ \\
\hline
\end{tabular}

AEs, adverse events; ADRs, adverse drug reactions; SAEs, severe adverse events; SADRs, severe adverse drug reactions

preoperative or intraoperative administration of bivalirudin $(P=0.036)$ were associated with an increased thrombocytopenia risk. After adjustment for multivariate analysis, only clinical presentation of SCAD $(P=0.002)$ and multiple culprit vessel $(P=0.010)$ were independently correlated with higher thrombocytopenia risk (Table 5).

In terms of bleeding risk, univariate analyses showed that clinical presentation of UA $(P=0.048)$ and higher post-procedure TIMI flow grade $(P=0.033)$ were associated with a reduced risk of bleeding, but history of 
Table 3 Detailed AEs and bivalirudin-related ADRs in System Organ Class (SOC)

\begin{tabular}{|c|c|c|c|c|c|c|c|c|}
\hline \multirow[t]{2}{*}{ Items } & \multicolumn{4}{|c|}{ AEs, No. (\%) } & \multicolumn{4}{|c|}{ Bivalirudin-related ADRs, No. (\%) } \\
\hline & Total & Mild & Moderate & Severe & Total & Mild & Moderate & Severe \\
\hline Total & $120(13.1)$ & $110(12.0)$ & $4(0.4)$ & $6(0.7)$ & $40(4.4)$ & $38(4.1)$ & $2(0.2)$ & $0(0.0)$ \\
\hline Gastrointestinal disorders & $38(4.1)$ & $35(3.8)$ & $2(0.2)$ & $1(0.1)$ & $10(1.1)$ & $8(0.9)$ & $2(0.2)$ & $0(0.0)$ \\
\hline Blood and lymphatic system disorders & $28(3.1)$ & $28(3.1)$ & $0(0.0)$ & $0(0.0)$ & $28(3.1)$ & $28(3.1)$ & $0(0.0)$ & $0(0.0)$ \\
\hline General disorders and administration site conditions & $27(2.9)$ & $27(2.9)$ & $0(0.0)$ & $0(0.0)$ & $0(0.0)$ & $0(0.0)$ & $0(0.0)$ & $0(0.0)$ \\
\hline Respiratory, thoracic, and mediastinal disorders & $24(2.6)$ & $22(2.4)$ & $1(0.1)$ & $1(0.1)$ & $0(0.0)$ & $0(0.0)$ & $0(0.0)$ & $0(0.0)$ \\
\hline Nervous system disorders & $15(1.6)$ & $11(1.2)$ & $2(0.2)$ & $2(0.2)$ & $0(0.0)$ & $0(0.0)$ & $0(0.0)$ & $0(0.0)$ \\
\hline Investigations & $14(1.5)$ & $12(1.3)$ & $1(0.1)$ & $1(0.1)$ & $2(0.2)$ & $2(0.2)$ & $0(0.0)$ & $0(0.0)$ \\
\hline Cardiac disorders & $12(1.3)$ & $10(1.1)$ & $0(0.0)$ & $2(0.2)$ & $1(0.1)$ & $1(0.1)$ & $0(0.0)$ & $0(0.0)$ \\
\hline Skin and subcutaneous tissue disorders & $8(0.9)$ & $8(0.9)$ & $0(0.0)$ & $0(0.0)$ & $0(0.0)$ & $0(0.0)$ & $0(0.0)$ & $0(0.0)$ \\
\hline Renal and urinary disorders & $7(0.8)$ & $7(0.8)$ & $0(0.0)$ & $0(0.0)$ & $0(0.0)$ & $0(0.0)$ & $0(0.0)$ & $0(0.0)$ \\
\hline Infections and infestations & $6(0.7)$ & $6(0.7)$ & $0(0.0)$ & $0(0.0)$ & $0(0.0)$ & $0(0.0)$ & $0(0.0)$ & $0(0.0)$ \\
\hline Metabolism and nutrition disorders & $5(0.5)$ & $5(0.5)$ & $0(0.0)$ & $0(0.0)$ & $0(0.0)$ & $0(0.0)$ & $0(0.0)$ & $0(0.0)$ \\
\hline Hepatobiliary disorders & $5(0.5)$ & $5(0.5)$ & $0(0.0)$ & $0(0.0)$ & $0(0.0)$ & $0(0.0)$ & $0(0.0)$ & $0(0.0)$ \\
\hline Psychiatric disorders & $5(0.5)$ & $5(0.5)$ & $0(0.0)$ & $0(0.0)$ & $0(0.0)$ & $0(0.0)$ & $0(0.0)$ & $0(0.0)$ \\
\hline Vascular disorders & $4(0.4)$ & $4(0.4)$ & $0(0.0)$ & $0(0.0)$ & $0(0.0)$ & $0(0.0)$ & $0(0.0)$ & $0(0.0)$ \\
\hline Musculoskeletal and connective tissue disorders & $3(0.3)$ & $3(0.3)$ & $0(0.0)$ & $0(0.0)$ & $0(0.0)$ & $0(0.0)$ & $0(0.0)$ & $0(0.0)$ \\
\hline Injury, poisoning and procedural complications & $2(0.2)$ & $2(0.2)$ & $0(0.0)$ & $0(0.0)$ & $1(0.1)$ & $1(0.1)$ & $0(0.0)$ & $0(0.0)$ \\
\hline Immune system disorders & $1(0.1)$ & $1(0.1)$ & $0(0.0)$ & $0(0.0)$ & $0(0.0)$ & $0(0.0)$ & $0(0.0)$ & $0(0.0)$ \\
\hline
\end{tabular}

AEs, adverse events; ADRs, adverse drug reactions

diabetes mellitus $(P=0.005)$ and CRUSADE high risk $(P=0.003)$ were linked to an increased risk of bleeding. Further multivariate analyses found that only history of diabetes mellitus $(P=0.007)$ and CRUSADE high risk $(P=0.016)$ were independent factor related to elevated risk of bleeding (Table 6).

\section{Discussion}

The efforts to investigate gender difference in CAD features or its treatment outcomes have never been stopped. For instance, it has been revealed that compared to male CAD patients, female CAD patients are often with older age at presentation, are accompanied with more comorbidities and severe disease condition [5, 17-23]. Furthermore, a growing number of researches observe that female patients underwent PCI exhibit a worse prognosis compared to male patients [24-29]. Notably, a recent meta-analysis analyzes 49 studies involving 1,032,828 patients reporting gender-specific outcomes in CAD patients underwent PCI, which discovers that major adverse cardiovascular event (MACE) and mortality are both increased, while revascularization rate is decreased in female patients compared to male patients [6]. Furthermore, it's also disclosed that gender-specific effect exists regarding different antiplatelet strategies [30, 31]. For example, the effect of P2Y12 inhibitor monotherapy after coronary revascularisation differs between females and males [30]. These evidenced point out the emphasis of PCI treated female patients.

Several top-level trials have reported the preeminence of bivalirudin over conventional heparin in terms of adverse events [9-14], for instance: a previous trial observed that net adverse clinical events (NACEs) $(9.2 \%$ vs. $12.1 \%)$ and major bleeding (4.9\% vs. $8.3 \%)$ were both attenuated by bivalirudin monotherapy compared with unfractionated heparin (UFH) plus a GP IIb/IIIa inhibitor in patients undergoing PCI [12]; another trial discovers that bivalirudin with provisional GP IIb/IIIa inhibitor reduces major bleeding rate versus heparin with planned GP IIb/IIIa inhibitor (2.4\% vs $4.1 \%$ ) in patients during PCI [11]. However, the studies focusing on female patients in this field are still finite. A trial discloses that bivalirudin achieves reduced incidences of 30 -day NACEs $(6.3 \%$ vs. 
Table 4 Analysis of factors related to ADRs

\begin{tabular}{|c|c|c|c|c|c|c|}
\hline \multirow[t]{2}{*}{ Items } & \multicolumn{2}{|c|}{$\begin{array}{l}\text { Bivalirudin-related } \\
\text { ADRs }\end{array}$} & \multicolumn{2}{|c|}{ Univariate } & \multicolumn{2}{|c|}{ Multivariate } \\
\hline & No (\%) & Yes (\%) & $P$ value & OR $(95 \% \mathrm{Cl})$ & $P$ value & OR $(95 \% \mathrm{Cl})$ \\
\hline Age & & & 0.146 & & - & \\
\hline$>75$ years & $217(93.9)$ & $14(6.1)$ & & $1.640(0.841-3.198)$ & & - \\
\hline$\leq 75$ years & $661(96.2)$ & $26(3.8)$ & & Reference & & - \\
\hline $\mathrm{BMI}$ & & & 0.284 & & - & \\
\hline$>28 \mathrm{~kg} / \mathrm{m}^{2}$ & $85(97.7)$ & $2(2.3)$ & & $0.455(0.108-1.920)$ & & - \\
\hline$\leq 28 \mathrm{~kg} / \mathrm{m}^{2}$ & $735(95.1)$ & $38(4.9)$ & & Reference & & - \\
\hline History of diabetes mellitus & & & 0.159 & & - & \\
\hline Yes & $259(94.2)$ & $16(5.8)$ & & 1.593 (0.833-3.049) & & - \\
\hline No & $619(96.3)$ & $24(3.7)$ & & Reference & & - \\
\hline History of allergy & & & 0.223 & & - & \\
\hline Yes & $98(93.3)$ & $7(6.7)$ & & $1.688(0.727-3.919)$ & & - \\
\hline No & $780(95.9)$ & $33(4.1)$ & & Reference & & - \\
\hline History of cardiac surgery & & & 0.268 & & - & \\
\hline Yes & $67(93.1)$ & $5(6.9)$ & & $1.729(0.656-4.560)$ & & - \\
\hline No & $811(95.9)$ & $35(4.1)$ & & Reference & & - \\
\hline History of renal function impairment & & & 0.095 & & - & \\
\hline Yes & $24(88.9)$ & $3(11.1)$ & & $2.885(0.831-10.015)$ & & - \\
\hline No & $854(95.8)$ & $37(4.2)$ & & Reference & & - \\
\hline History of critical respiratory disease & & & 0.887 & & - & \\
\hline Yes & $19(95.0)$ & $1(5.0)$ & & $1.159(0.151-8.883)$ & & - \\
\hline No & $859(95.7)$ & $39(4.3)$ & & Reference & & - \\
\hline Clinical presentation-UA & & & 0.006 & & 0.088 & \\
\hline Yes & $353(98.1)$ & $7(1.9)$ & & $0.315(0.138-0.721)$ & & $0.463(0.191-1.122)$ \\
\hline No & $525(94.1)$ & $33(5.9)$ & & Reference & & Reference \\
\hline Clinical presentation-STEMI & & & 0.371 & & - & \\
\hline Yes & $312(94.8)$ & $17(5.2)$ & & $1.341(0.706-2.548)$ & & - \\
\hline No & $566(96.1)$ & $23(3.9)$ & & Reference & & - \\
\hline Clinical presentation-NSTMI & & & 0.773 & & - & \\
\hline Yes & $124(96.1)$ & $5(3.9)$ & & $0.869(0.334-2.260)$ & & - \\
\hline No & $754(95.6)$ & $35(4.4)$ & & Reference & & - \\
\hline Clinical presentation-SCAD & & & 0.001 & & 0.004 & \\
\hline Yes & $88(88.9)$ & $11(11.1)$ & & $3.405(1.644-7.053)$ & & $3.191(1.446-7.044)$ \\
\hline No & $790(96.5)$ & $29(3.5)$ & & Reference & & Reference \\
\hline CRUSADE risk stratification & & & 0.005 & & 0.031 & \\
\hline High risk & $229(92.3)$ & $19(7.7)$ & & $2.505(1.323-4.744)$ & & $2.075(1.070-4.024)$ \\
\hline Non-high risk & $634(96.8)$ & $21(3.2)$ & & Reference & & Reference \\
\hline Operative timing & & & 0.209 & & - & \\
\hline Elective operation & $548(96.3)$ & $21(3.7)$ & & $0.666(0.353-1.256)$ & & - \\
\hline Emergency operation & $330(94.6)$ & $19(5.4)$ & & Reference & & - \\
\hline Types of coronary interventional therapy & & & 0.997 & & - & \\
\hline Stent implantation & $834(95.6)$ & $38(4.4)$ & & $1.002(0.234-4.290)$ & & - \\
\hline Others & 44(95.7) & $2(4.3)$ & & Reference & & - \\
\hline Types of stents & & & 0.659 & & - & \\
\hline Drug stent & $823(95.7)$ & $37(4.3)$ & & $0.629(0.081-4.915)$ & & - \\
\hline Others & $14(93.3)$ & $1(6.7)$ & & Reference & & - \\
\hline Arterial access & & & 0.564 & & - & \\
\hline Radial artery & $812(95.8)$ & $36(4.2)$ & & $0.732(0.253-2.118)$ & & - \\
\hline
\end{tabular}


Table 4 (continued)

\begin{tabular}{|c|c|c|c|c|c|c|}
\hline \multirow[t]{2}{*}{ Items } & \multicolumn{2}{|c|}{$\begin{array}{l}\text { Bivalirudin-related } \\
\text { ADRs }\end{array}$} & \multicolumn{2}{|c|}{ Univariate } & \multicolumn{2}{|c|}{ Multivariate } \\
\hline & No (\%) & Yes (\%) & $P$ value & OR $(95 \% \mathrm{Cl})$ & $P$ value & OR $(95 \% \mathrm{Cl})$ \\
\hline Others & $66(94.3)$ & $4(5.7)$ & & Reference & & - \\
\hline Culprit vessel & & & 0.048 & & 0.019 & \\
\hline Multiple & $189(93.1)$ & $14(6.9)$ & & $1.963(1.005-3.834)$ & & $2.328(1.146-4.728)$ \\
\hline Single & $689(96.4)$ & $26(3.6)$ & & Reference & & Reference \\
\hline Administration of bivalirudin-preoperative or intraoperative & & & 0.026 & & 0.116 & \\
\hline Yes & $27(87.1)$ & $4(12.9)$ & & $3.502(1.164-10.539)$ & & $2.522(0.796-7.990)$ \\
\hline No & $851(95.9)$ & $36(4.1)$ & & Reference & & Reference \\
\hline Administration of bivalirudin-postoperative $\leq 4 \mathrm{~h}$ & & & 0.383 & & - & \\
\hline Yes & $747(95.9)$ & $32(4.1)$ & & $0.701(0.316-1.556)$ & & - \\
\hline No & $131(94.2)$ & $8(5.8)$ & & Reference & & - \\
\hline Administration of bivalirudin-postoperative $>4 \mathrm{~h}$ & & & 0.724 & & - & \\
\hline Yes & $104(96.3)$ & $4(3.7)$ & & $0.827(0.288-2.370)$ & & - \\
\hline No & $774(95.6)$ & $36(4.4)$ & & Reference & & - \\
\hline Combined with GP IIb/Illa inhibitors & & & 0.265 & & - & \\
\hline Yes & $631(95.2)$ & $32(4.8)$ & & $1.566(0.712-3.445)$ & & - \\
\hline No & $247(96.9)$ & $8(3.1)$ & & Reference & & - \\
\hline TIMI flow grade (pre-procedure) & & & 0.491 & & - & \\
\hline $2-3$ & $507(96.0)$ & $21(4.0)$ & & $0.800(0.424-1.510)$ & & - \\
\hline $0-1$ & $367(95.1)$ & $19(4.9)$ & & Reference & & - \\
\hline TIMI flow grade (post-procedure) & & & 0.284 & & - & \\
\hline $2-3$ & $870(95.7)$ & $39(4.3)$ & & $0.314(0.038-2.613)$ & & - \\
\hline $0-1$ & $7(87.5)$ & $1(12.5)$ & & Reference & & - \\
\hline
\end{tabular}

Bold value means statistically significant

ADRs, adverse drug reactions; $\mathrm{OR}$, odds ratio; $\mathrm{Cl}$, confidence interval; $\mathrm{BMI}$, body mass indexes; $\mathrm{UA}$, unstable angina; STEMI, ST-segment elevation myocardial infarction; NSTMI, non-ST-segment elevation myocardial infarction; SCAD, spontaneous coronary artery dissection; CRUSADE, Can Rapid Risk Stratification of Unstable Angina Patients Suppress Adverse Outcomes with Early Implementation of the ACC/AHA Guidelines; GP, glycoprotein; TIMI, thrombolysis in myocardial infarction

$21.5 \%)$, any bleeding (2.4\% vs. $12.8 \%$ ) and BARC $2-5$ type bleeding (1.6\% vs. $7.2 \%)$ compared to heparin with or without tirofiban in female patients undergoing PCI [32]. Nevertheless, there are limited reports regarding the AEs or ADRs of bivalirudin during PCI in real-world condition, not to mention that bivalirudin lacks data in Chinese female patients. In our present study, we observed that the incidence of AEs, SAEs, bivalirudin-related ADRs and bivalirudin-related SADRs was $13.1 \%, 0.8 \%$, $4.4 \%$ and $0.3 \%$, respectively, furthermore, $2.9 \%$ and $1.4 \%$ patients experienced thrombocytopenia and bleeding, in Chinese female patients undergoing PCI with bivalirudin as anticoagulant. Our data of AEs incidence was within the range of that in previous studies, which did not assess the bivalirudin-related ADRs incidence, therefore, it could not be referred. Interestingly, it was observed that thrombocytopenia and bleeding incidences by bivalirudin were relatively less in our present study compared to those published data previously, the possible explanations are: (1) Chinese patients may have less complications (such as obesity, hyperlipidemia, diabetes, kidney diseases), which is relates to less thrombocytopenia and bleeding risk; (2) Different study design, observational period and so on might influence the data.

Subsequently, in our study, it was found that clinical manifestation of SCAD, CRUSADE high risk, multiple culprit vessel was independently correlated with higher risk of bivalirudin-related ADRs. Several possible explanations are listed as follows: (1) PCI is selectively proposed for SCAD treatment with an increased risk of 
Table 5 Analysis of factors related to thrombocytopenia

\begin{tabular}{|c|c|c|c|c|c|c|}
\hline \multirow[t]{2}{*}{ Items } & \multicolumn{2}{|c|}{ Thrombocytopenia } & \multicolumn{2}{|c|}{ Univariate } & \multicolumn{2}{|c|}{ Multivariate } \\
\hline & No (\%) & Yes (\%) & $P$ value & OR $(95 \% \mathrm{Cl})$ & $P$ value & OR $(95 \% \mathrm{Cl})$ \\
\hline Age & & & 0.324 & & - & \\
\hline$>75$ years & $222(96.1)$ & $9(3.9)$ & & $1.507(0.667-3.402)$ & & - \\
\hline$\leq 75$ years & $669(97.4)$ & $18(2.6)$ & & Reference & & - \\
\hline BMI & & & 0.285 & & - & \\
\hline$>28 \mathrm{~kg} / \mathrm{m}^{2}$ & $86(98.9)$ & $1(1.1)$ & & $0.334(0.045-2.493)$ & & - \\
\hline$\leq 28 \mathrm{~kg} / \mathrm{m}^{2}$ & $747(96.6)$ & $26(3.4)$ & & Reference & & - \\
\hline History of diabetes mellitus & & & 0.970 & & - & \\
\hline Yes & $267(97.1)$ & $8(2.9)$ & & $0.984(0.425-2.276)$ & & - \\
\hline No & $624(97.0)$ & $19(3.0)$ & & Reference & & - \\
\hline History of allergy & & & 0.247 & & - & \\
\hline Yes & $100(95.2)$ & $5(4.8)$ & & $1.798(0.666-4.853)$ & & - \\
\hline No & $791(97.3)$ & $22(2.7)$ & & Reference & & - \\
\hline History of cardiac surgery & & & 0.181 & & - & \\
\hline Yes & $68(94.4)$ & $4(5.6)$ & & 2.105 (0.708-6.262) & & - \\
\hline No & $823(97.3)$ & $23(2.7)$ & & Reference & & - \\
\hline History of renal function impairment & & & 0.181 & & - & \\
\hline Yes & $25(92.6)$ & $2(7.4)$ & & $2.771(0.622-12.347)$ & & - \\
\hline No & $866(97.2)$ & $25(2.8)$ & & Reference & & - \\
\hline History of critical respiratory disease & & & 0.587 & & - & \\
\hline Yes & $19(95.0)$ & $1(5.0)$ & & $1.765(0.228-13.689)$ & & - \\
\hline No & $872(97.1)$ & $26(2.9)$ & & Reference & & - \\
\hline Clinical presentation-UA & & & 0.032 & & 0.187 & \\
\hline Yes & $355(98.6)$ & $5(1.4)$ & & $0.343(0.129-0.915)$ & & $0.492(0.171-1.412)$ \\
\hline No & $536(96.1)$ & $22(3.9)$ & & Reference & & Reference \\
\hline Clinical presentation-STEMI & & & 0.895 & & - & \\
\hline Yes & $319(97.0)$ & $10(3.0)$ & & $1.055(0.477-2.331)$ & & - \\
\hline No & $572(97.1)$ & $17(2.9)$ & & Reference & & - \\
\hline Clinical presentation-NSTMI & & & 0.656 & & - & \\
\hline Yes & $126(97.7)$ & $3(2.3)$ & & $0.759(0.225-2.558)$ & & - \\
\hline No & $765(97.0)$ & $24(3.0)$ & & Reference & & - \\
\hline Clinical presentation-SCAD & & & $<0.001$ & & 0.002 & \\
\hline Yes & $90(90.9)$ & $9(9.1)$ & & $4.450(1.942-10.198)$ & & $4.388(1.754-10.981)$ \\
\hline No & $801(97.8)$ & $18(2.2)$ & & Reference & & Reference \\
\hline CRUSADE risk stratification & & & 0.122 & & - & \\
\hline High risk & $237(95.6)$ & $11(4.4)$ & & $1.854(0.848-4.052)$ & & - \\
\hline No high risk & $639(97.6)$ & $16(2.4)$ & & Reference & & - \\
\hline Operative timing & & & 0.767 & & - & \\
\hline Elective operation & $553(97.2)$ & $16(2.8)$ & & $0.889(0.408-1.938)$ & & - \\
\hline Emergency operation & $338(96.8)$ & $11(3.2)$ & & Reference & & - \\
\hline Types of coronary interventional therapy & & & 0.665 & & - & \\
\hline Stent implantation & $846(97.0)$ & $26(3.0)$ & & $0.672(0.111-4.056)$ & & - \\
\hline Others & $45(97.8)$ & $1(2.2)$ & & Reference & & - \\
\hline Types of stents & & & 0.410 & & - & \\
\hline Drug stent & $835(97.1)$ & $25(2.9)$ & & $0.419(0.053-3.313)$ & & - \\
\hline Others & $14(93.3)$ & $1(6.7)$ & & Reference & & - \\
\hline Arterial access & & & 0.163 & & - & \\
\hline Radial artery & $825(97.3)$ & $23(2.7)$ & & $0.460(0.155-1.370)$ & & - \\
\hline Others & $66(94.3)$ & $4(5.7)$ & & Reference & & - \\
\hline
\end{tabular}


Table 5 (continued)

\begin{tabular}{|c|c|c|c|c|c|c|}
\hline \multirow[t]{2}{*}{ Items } & \multicolumn{2}{|c|}{ Thrombocytopenia } & \multicolumn{2}{|c|}{ Univariate } & \multicolumn{2}{|c|}{ Multivariate } \\
\hline & No (\%) & Yes (\%) & $P$ value & OR $(95 \% \mathrm{Cl})$ & $P$ value & OR $(95 \% \mathrm{Cl})$ \\
\hline Culprit vessel & & & 0.022 & & 0.010 & \\
\hline Multiple & $192(94.6)$ & $11(5.4)$ & & $2.503(1.143-5.483)$ & & $2.974(1.302-6.792)$ \\
\hline Single & $699(97.8)$ & $16(2.2)$ & & Reference & & Reference \\
\hline Administration of bivalirudin-preoperative or intraoperative & & & 0.036 & & 0.081 & \\
\hline Yes & $28(90.3)$ & $3(9.7)$ & & $3.853(1.095-13.553)$ & & $3.220(0.867-11.953)$ \\
\hline No & $863(97.3)$ & $24(2.7)$ & & Reference & & Reference \\
\hline Administration of bivalirudin-postoperative $\leq 4 \mathrm{~h}$ & & & 0.962 & & - & \\
\hline Yes & $756(97.0)$ & $23(3.0)$ & & $1.027(0.350-3.016)$ & & - \\
\hline No & $135(97.1)$ & $4(2.9)$ & & Reference & & - \\
\hline Administration of bivalirudin-postoperative $>4 \mathrm{~h}$ & & & 0.216 & & - & \\
\hline Yes & $107(99.1)$ & $1(0.9)$ & & $0.282(0.038-2.098)$ & & - \\
\hline No & $784(96.8)$ & $26(3.2)$ & & Reference & & - \\
\hline Combined with GP IIb/IIla inhibitors & & & 0.281 & & - & \\
\hline Yes & $641(96.7)$ & $22(3.3)$ & & $1.716(0.643-4.581)$ & & - \\
\hline No & $250(98.0)$ & $5(2.0)$ & & Reference & & - \\
\hline TIMI flow grade (pre-procedure) & & & 0.074 & & - & \\
\hline $2-3$ & $517(97.9)$ & $11(2.1)$ & & $0.492(0.226-1.072)$ & & - \\
\hline $0-1$ & $370(95.9)$ & $16(4.1)$ & & Reference & & - \\
\hline TIMI flow grade (post-procedure) & & & 0.999 & & - & \\
\hline $2-3$ & $882(97.0)$ & $27(3.0)$ & & - & & - \\
\hline $0-1$ & $8(100.0)$ & $0(0.0)$ & & - & & - \\
\hline
\end{tabular}

Bold value means statistically significant

OR, odds ratio; Cl, confidence interval; BMI, body mass indexes; UA, unstable angina; STEMI, ST-segment elevation myocardial infarction; NSTMI, non-ST-segment elevation myocardial infarction; SCAD, spontaneous coronary artery dissection; CRUSADE, Can Rapid Risk Stratification of Unstable Angina Patients Suppress Adverse Outcomes with Early Implementation of the ACC/AHA Guidelines; GP, glycoprotein; TIMI, thrombolysis in myocardial infarction

complications such as Iatrogenic dissection, acute vascular occlusion and hematoma extending, is therefore correlated with higher ADRs [33]; (2) CRUSADE high risk and diabetes mellitus are well-known risk factors for bleeding during PCI, therefore relates to increased ADRs and bleeding risk; (3) multiple culprit vessel indicates more severe disease conditions leading to higher ADRs.

Some limitations of the current study needed to be addressed: firstly, due to the total bivalirudin-related ADRs incidence was low, the sample size of nearly one thousand might not be sufficient to make a confirmative conclusion, therefore future larger sample-sized study was needed; secondly, the low ADRs, thrombocytopenia and bleeding incidences also reduced the statistical power of logistic analyses.

\section{Conclusion}

To be conclusive, bivalirudin is well tolerated with low ADRs, thrombocytopenia and bleeding incidences in female patients undergoing PCI. 
Table 6 Analysis of factors related to bleeding

\begin{tabular}{|c|c|c|c|c|c|c|}
\hline \multirow[t]{2}{*}{ Items } & \multicolumn{2}{|l|}{ Bleeding } & \multicolumn{2}{|c|}{ Univariate } & \multicolumn{2}{|c|}{ Multivariate } \\
\hline & No (\%) & Yes (\%) & $P$ value & OR $(95 \% \mathrm{Cl})$ & $P$ value & OR $(95 \% \mathrm{Cl})$ \\
\hline Age & & & 0.273 & & - & \\
\hline$>75$ years & $226(97.8)$ & $5(2.2)$ & & $1.878(0.608-5.798)$ & & - \\
\hline$\leq 75$ years & $679(98.8)$ & $8(1.2)$ & & Reference & & - \\
\hline BMI & & & 0.997 & & - & \\
\hline$>28 \mathrm{~kg} / \mathrm{m}^{2}$ & $87(100.0)$ & $0(0.0)$ & & - & & - \\
\hline$\leq 28 \mathrm{~kg} / \mathrm{m}^{2}$ & $760(98.3)$ & $13(1.7)$ & & - & & - \\
\hline History of diabetes mellitus & & & 0.005 & & 0.007 & \\
\hline Yes & $266(96.7)$ & $9(3.3)$ & & $5.405(1.650-17.704)$ & & $5.227(1.562-17.495)$ \\
\hline No & $639(99.4)$ & $4(0.6)$ & & Reference & & Reference \\
\hline History of allergy & & & 0.654 & & - & \\
\hline Yes & $103(98.1)$ & $2(1.9)$ & & $1.416(0.309-6.476)$ & & - \\
\hline No & $802(98.6)$ & $11(1.4)$ & & Reference & & - \\
\hline History of cardiac surgery & & & 0.984 & & - & \\
\hline Yes & $71(98.6)$ & $1(1.4)$ & & $0.979(0.125-7.637)$ & & - \\
\hline No & 834 (98.6) & $12(1.4)$ & & Reference & & - \\
\hline History of renal function impairment & & & 0.328 & & - & \\
\hline Yes & $26(96.3)$ & $1(3.7)$ & & $2.817(0.353-22.482)$ & & - \\
\hline No & $879(98.7)$ & $12(1.3)$ & & Reference & & - \\
\hline History of critical respiratory disease & & & 0.998 & & - & \\
\hline Yes & $20(100.0)$ & $0(0.0)$ & & - & & - \\
\hline No & $885(98.6)$ & $13(1.4)$ & & - & & - \\
\hline Clinical presentation-UA & & & 0.048 & & 0.103 & \\
\hline Yes & $359(99.7)$ & $1(0.3)$ & & $0.127(0.016-0.979)$ & & $0.178(0.022-1.420)$ \\
\hline No & $546(97.8)$ & $12(2.2)$ & & Reference & & Reference \\
\hline Clinical presentation-STEMI & & & 0.182 & & - & \\
\hline Yes & $322(97.9)$ & $7(2.1)$ & & $2.112(0.704-6.339)$ & & - \\
\hline No & $583(99.0)$ & $6(1.0)$ & & Reference & & - \\
\hline Clinical presentation-NSTMI & & & 0.889 & & - & \\
\hline Yes & $127(98.4)$ & $2(1.6)$ & & $1.114(0.244-5.084)$ & & - \\
\hline No & $778(98.6)$ & $11(1.4)$ & & Reference & & - \\
\hline Clinical presentation-SCAD & & & 0.164 & & - & \\
\hline Yes & $96(97.0)$ & $3(3.0)$ & & $2.528(0.684-9.346)$ & & - \\
\hline No & $809(98.8)$ & $10(1.2)$ & & Reference & & - \\
\hline CRUSADE risk stratification & & & 0.003 & & 0.016 & \\
\hline High risk & $239(96.4)$ & $9(3.6)$ & & $6.129(1.870-20.087)$ & & $4.475(1.323-15.134)$ \\
\hline No high risk & $651(99.4)$ & $4(0.6)$ & & Reference & & Reference \\
\hline Operative timing & & & 0.090 & & - & \\
\hline Elective operation & $564(99.1)$ & $5(0.9)$ & & $0.378(0.123-1.164)$ & & - \\
\hline Emergency operation & $341(97.7)$ & $8(2.3)$ & & Reference & & - \\
\hline Types of coronary interventional therapy & & & 0.658 & & - & \\
\hline Stent implantation & 860 (98.6) & $12(1.4)$ & & $0.628(0.080-4.936)$ & & - \\
\hline Others & $45(97.8)$ & $1(2.2)$ & & Reference & & - \\
\hline Types ofstents & & & 0.999 & & - & \\
\hline Drug stent & $848(98.6)$ & $12(1.4)$ & & - & & - \\
\hline Others & $15(100.0)$ & $0(0.0)$ & & - & & - \\
\hline Arterial access & & & 0.997 & & - & \\
\hline Radial artery & $835(98.5)$ & $13(1.5)$ & & - & & - \\
\hline Others & $70(100.0)$ & $0(0.0)$ & & - & & - \\
\hline Culprit vessel & & & 0.559 & & - & \\
\hline Multiple & $201(99.0)$ & $2(1.0)$ & & $0.637(0.140-2.896)$ & & - \\
\hline Single & 704 (98.5) & $11(1.5)$ & & Reference & & - \\
\hline
\end{tabular}


Table 6 (continued)

\begin{tabular}{|c|c|c|c|c|c|c|}
\hline \multirow[t]{2}{*}{ Items } & \multicolumn{2}{|l|}{ Bleeding } & \multicolumn{2}{|c|}{ Univariate } & \multicolumn{2}{|c|}{ Multivariate } \\
\hline & No (\%) & Yes (\%) & $P$ value & OR $(95 \% \mathrm{Cl})$ & $P$ value & OR $(95 \% \mathrm{Cl})$ \\
\hline Administration of bivalirudin-preoperative or intraoperative & & & 0.401 & & - & \\
\hline Yes & $30(96.8)$ & $1(3.2)$ & & $2.431(0.306-19.304)$ & & - \\
\hline No & $875(98.6)$ & $12(1.4)$ & & Reference & & - \\
\hline Administration of bivalirudin-postoperative $\leq 4 \mathrm{~h}$ & & & 0.126 & & - & \\
\hline Yes & $770(98.8)$ & $9(1.2)$ & & $0.394(0.120-1.299)$ & & - \\
\hline No & $135(97.1)$ & $4(2.9)$ & & Reference & & - \\
\hline Administration of bivalirudin-postoperative $>4 \mathrm{~h}$ & & & 0.215 & & - & \\
\hline Yes & $105(97.2)$ & $3(2.8)$ & & $2.286(0.619-8.439)$ & & - \\
\hline No & $800(98.8)$ & $10(1.2)$ & & Reference & & - \\
\hline Combined with GP IIb/IIla inhibitors & & & 0.326 & & - & \\
\hline Yes & $652(98.3)$ & $11(1.7)$ & & $2.134(0.470-9.696)$ & & - \\
\hline No & $253(99.2)$ & $2(0.8)$ & & Reference & & - \\
\hline TIMI flow grade (pre-procedure) & & & 0.173 & & - & \\
\hline $2-3$ & $518(98.1)$ & $10(1.9)$ & & $2.465(0.674-9.016)$ & & - \\
\hline $0-1$ & $383(99.2)$ & $3(0.8)$ & & Reference & & - \\
\hline TIMI flow grade(post-procedure) & & & 0.033 & & 0.069 & \\
\hline $2-3$ & $897(98.7)$ & $12(1.3)$ & & $0.094(0.011-0.821)$ & & $0.105(0.009-1.187)$ \\
\hline $0-1$ & $7(87.5)$ & $1(12.5)$ & & Reference & & Reference \\
\hline
\end{tabular}

Bold value means statistically significant

OR, odds ratio; $\mathrm{Cl}$, confidence interval; BMI, body mass indexes; UA, unstable angina; STEMI, ST-segment elevation myocardial infarction; NSTMI, non-ST-segment elevation myocardial infarction; SCAD, spontaneous coronary artery dissection; CRUSADE, Can Rapid Risk Stratification of Unstable Angina Patients Suppress Adverse Outcomes with Early Implementation of the ACC/AHA Guidelines; GP, glycoprotein; TIMI, thrombolysis in myocardial infarction

\section{Abbreviations}

PCI: Percutaneous coronary intervention; DES: Drug-eluting stents; CAD: Coronary artery disease; AEs: Adverse events; ADRs: Adverse drug reactions; BMI: Body mass indexes; UA: Unstable angina; STEMI: ST-segment elevation myocardial infarction; NSTMI: Non-ST-segment elevation myocardial infarction; SCAD: Spontaneous coronary artery dissection; GP: Glycoprotein; TIMI: Thrombolysis in myocardial infarction; SOC: Systematic organ classification; PT: Preferred term; MedDRA: Medical dictionary for regulatory activities; SAEs: Severe adverse events; SADRs: Severe adverse drug reactions; UA: Unstable angina; NACEs: Net adverse clinical events; UFH: Unfractionated heparin.

\section{Acknowledgements}

Not applicable.

\section{Authors' contributions}

FW and XL made substantial contributions to the design of the present study. Data acquisition and interpretation was performed by FW, XL, HR, QT, CZ, YW and JX. YW and JX critically revised the manuscript for important intellectual content. All authors approved the final version of the manuscript. All authors agree to be accountable for all aspects of the work in ensuring that questions related to the accuracy or integrity of the work are appropriately investigated and resolved. All authors read and approved the final manuscript.

\section{Funding}

This study was supported by Construction Plan of the Superior Science and Technology Innovation Team of Jiangxi Province (No.20181BCB24013), Senior Medical Talents Program of Chongqing for Young and Middle-aged (2017HBRC014) and Sports Science Research Project of Chongqing Municipal Bureau of Sports (D202001). These funders were used to pay for the costs incurred in the study process, including study design, data collection and analysis, as well as article publication costs.

\section{Availability of data and material}

All relevant data is presented in the manuscript and supporting material.

\section{Declarations}

Ethics approval and consent to participate

The trial was conducted in accordance with the Declaration of Helsinki, and was approved by the Ethics Committee of the Chongqing University Central Hospital and all patients provided the written informed consents.

\section{Consent for publication}

Not applicable.

\section{Competing interests}

The authors declare that they have no competing interests.

\section{Author details}

${ }^{1}$ Department of Cardiovascular Medicine II, Xuchang Central Hospital, 30 Huatuo Road, Xuchang 461001, Henan Province, China. ${ }^{2}$ Department of Cardiology, The First Hospital of Zhangjiakou, No.6 Chapel Lane, Xinhua Front Street, Qiaoxi District, Zhangjiakou 075061, Hunan, China. ${ }^{3}$ Department of Cardio-Thoracic Surgery, ZhouKou Center Hospistal of Henan Province, East Section of Renmin Road, Zhoukou 466699, Henan, China. ${ }^{4}$ Department of Cardiology, The First Affiliated Hospital of Henan University, 357 Ximen Dajie, Kaifeng 475004, Henan, China. ${ }^{5}$ Department of Cardiovascular Medicine, Zhejiang Lvcheng Cardiovascular Hospital, 409 Gudun Road, Wenxin Street, Xihu District, Hangzhou 310011, Zhejiang, China. ${ }^{6}$ Department of Cardiovascular Medicine, The Second Affiliated Hospital of Nanchang University, 1 Minde Road, Donghu District, Nanchang 330006, China. ${ }^{7}$ Department of Cardiovascular Medicine, Chongqing University Central Hospital, No. 1 Jiankang Road, Yuzhong District, Chongqing 400014, China.

Received: 11 August 2021 Accepted: 12 January 2022

Published online: 17 February 2022 


\section{References}

1. Teoh Z, Al-Lamee RK. COURAGE, ORBITA, and ISCHEMIA: percutaneous coronary intervention for stable coronary artery disease. Interv Cardiol Clin. 2020;9(4):469-82.

2. Burzotta F, Lassen JF, Banning AP, Lefevre T, Hildick-Smith D, Chieffo A, Darremont $\mathrm{O}$, Pan M, Chatzizisis YS, Albiero R, et al. Percutaneous coronary intervention in left main coronary artery disease: the 13th consensus document from the European Bifurcation Club. Eurolntervention. 2018;14(1):112-20.

3. Calabro P, Niccoli G, Gragnano F, Grove EL, Vergallo R, Mikhailidis DP, Patti G, Spaccarotella C, Katsiki N, Masiero G, et al. Are we ready for a gender-specific approach in interventional cardiology? Int J Cardiol. 2019;286:226-33.

4. Mehilli J, Presbitero P. Coronary artery disease and acute coronary syndrome in women. Heart. 2020;106(7):487-92

5. Greco A, Capodanno D. Differences in coronary artery disease and outcomes of percutaneous coronary intervention with drug-eluting stents in women and men. Expert Rev Cardiovasc Ther. 2021;19(4):301-12.

6. Guo Y, Yin F, Fan C, Wang Z. Gender difference in clinical outcomes of the patients with coronary artery disease after percutaneous coronary intervention: A systematic review and meta-analysis. Medicine (Baltimore). 2018:97(30):e11644.

7. Anand SX, Kim MC, Kamran M, Sharma SK, Kini AS, Fareed J, Hoppensteadt DA, Carbon F, Cavusoglu E, Varon D, et al. Comparison of platelet function and morphology in patients undergoing percutaneous coronary intervention receiving bivalirudin versus unfractionated heparin versus clopidogrel pretreatment and bivalirudin. Am J Cardiol. 2007;100(3):417-24.

8. Weitz Jl, Hudoba M, Massel D, Maraganore J, Hirsh J. Clot-bound thrombin is protected from inhibition by heparin-antithrombin III but is susceptible to inactivation by antithrombin III-independent inhibitors. J Clin Invest. 1990;86(2):385-91.

9. Stone GW, McLaurin BT, Cox DA, Bertrand ME, Lincoff AM, Moses JW, White HD, Pocock SJ, Ware JH, Feit F, et al. Bivalirudin for patients with acute coronary syndromes. N Engl J Med. 2006;355(21):2203-16.

10. Bittl JA, Chaitman BR, Feit F, Kimball W, Topol EJ. Bivalirudin versus heparin during coronary angioplasty for unstable or postinfarction angina: Final report reanalysis of the Bivalirudin Angioplasty Study. Am Heart J. 2001;142(6):952-9.

11. Lincoff AM, Bittl JA, Harrington RA, Feit F, Kleiman NS, Jackman JD, Sarembock IJ, Cohen DJ, Spriggs D, Ebrahimi R, et al. Bivalirudin and provisional glycoprotein Ilb/Illa blockade compared with heparin and planned glycoprotein IIb/Illa blockade during percutaneous coronary intervention: REPLACE-2 randomized trial. JAMA. 2003;289(7):853-63.

12. Stone GW, Witzenbichler B, Guagliumi G, Peruga JZ, Brodie BR, Dudek D, Kornowski R, Hartmann F, Gersh BJ, Pocock SJ, et al. Bivalirudin during primary $\mathrm{PCl}$ in acute myocardial infarction. N Engl J Med. 2008;358(21):2218-30.

13. Mehran R, Lansky AJ, Witzenbichler B, Guagliumi G, Peruga JZ, Brodie BR, Dudek D, Kornowski R, Hartmann F, Gersh BJ, et al. Bivalirudin in patients undergoing primary angioplasty for acute myocardial infarction (HORIZONS-AMI): 1-year results of a randomised controlled trial. Lancet. 2009:374(9696):1149-59.

14. Stone GW: HORIZONS- AMI:Two-Year Follow-up from a Prospective, Randomized Trial of Heparin Plus Glycoprotein IIb/llla Inhibitors vs. Bivalirudin and Paclitaxel-Eluting vs. Bare-Metal Stents in STEMI. In: TCT2009 Conference: 25 September 2009: San Francisco; 2009.

15. Subherwal S, Bach RG, Chen AY, Gage BF, Rao SV, Newby LK, Wang TY, Gibler WB, Ohman EM, Roe MT, et al. Baseline risk of major bleeding in non-STsegment-elevation myocardial infarction: the CRUSADE (Can Rapid risk stratification of Unstable angina patients Suppress ADverse outcomes with Early implementation of the ACC/AHA Guidelines) Bleeding Score. Circulation. 2009;119(14):1873-82.

16. Mehran R, Rao SV, Bhatt DL, Gibson CM, Caixeta A, Eikelboom J, Kaul S, Wiviott SD, Menon V, Nikolsky E, et al. Standardized bleeding definitions for cardiovascular clinical trials: a consensus report from the bleeding academic research consortium. Circulation. 2011;123(23):2736-47.

17. Towfighi A, Zheng L, Ovbiagele B. Sex-specific trends in midlife coronary heart disease risk and prevalence. Arch Intern Med. 2009;169(19):1762-6.

18. Ahmed B, Dauerman HL. Women, bleeding, and coronary intervention. Circulation. 2013;127(5):641-9.

19. Sheifer SE, Canos MR, Weinfurt KP, Arora UK, Mendelsohn FO, Gersh BJ, Weissman NJ. Sex differences in coronary artery size assessed by intravascular ultrasound. Am Heart J. 2000;139(4):649-53.
20. Herity NA, Lo S, Lee DP, Ward MR, Filardo SD, Yock PG, Fitzgerald PJ, Hunt SA, Yeung AC. Effect of a change in gender on coronary arterial size: a longitudinal intravascular ultrasound study in transplanted hearts. J Am Coll Cardiol. 2003;41(9):1539-46.

21. Vaccarino V, Badimon L, Corti R, de Wit C, Dorobantu M, Hall A, Koller A, Marzilli M, Pries A, Bugiardini R, et al. Ischaemic heart disease in women: are there sex differences in pathophysiology and risk factors? Position paper from the working group on coronary pathophysiology and microcirculation of the European Society of Cardiology. Cardiovasc Res. 2011;90(1):9-17.

22. Saeedi P, Petersohn I, Salpea P, Malanda B, Karuranga S, Unwin N, Colagiuri S, Guariguata L, Motala AA, Ogurtsova Ket al: Global and regional diabetes prevalence estimates for 2019 and projections for 2030 and 2045: Results from the International Diabetes Federation Diabetes Atlas, 9(th) edition. Diabetes Res Clin Pract 2019, 157:107843.

23. Buja P, D'Amico G, Facchin M, Barioli A, Napodano M, Capodanno D, Musumeci G, Frigo AC, Saia F, Menozzi A, et al. Gender-related differences of diabetic patients undergoing percutaneous coronary intervention with drug-eluting stents: a real-life multicenter experience. Int J Cardiol. 2013;168(1):139-43.

24. Birkemeyer R, Schneider H, Rillig A, Ebeling J, Akin I, Kische S, Paranskaya L, Jung W, Ince $\mathrm{H}$, Nienaber CA. Do gender differences in primary PCI mortality represent a different adherence to guideline recommended therapy? A multicenter observation. BMC Cardiovasc Disord. 2014;14:71.

25. Toyota T, Furukawa Y, Ehara N, Funakoshi S, Morimoto T, Kaji S, Nakagawa Y, Kadota K, Iwabuchi M, Shiomi H, et al. Sex-based differences in clinical practice and outcomes for Japanese patients with acute myocardial infarction undergoing primary percutaneous coronary intervention. Circ J. 2013;77(6):1508-17.

26. Pain TE, Jones DA, Rathod KS, Gallagher SM, Knight CJ, Mathur A, Rothman $M T$, Jain AK, Wragg A. Influence of female sex on long-term mortality after acute coronary syndromes treated by percutaneous coronary intervention: a cohort study of 7304 patients. Coron Artery Dis. 2013;24(3):183-90.

27. Numasawa Y, Inohara T, Ishii H, Kuno T, Kodaira M, Kohsaka S, Fujii K, Uemura S, Amano T, Kadota K, et al. Comparison of outcomes of women versus men with non-ST-elevation acute coronary syndromes undergoing percutaneous coronary intervention (from the Japanese nationwide registry). Am J Cardiol. 2017;119(6):826-31.

28. Jarrah MI, Hammoudeh AJ, Al-Natour DB, Khader YS, Tabbalat RA, Alhaddad IA, Kullab SM. Gender differences in risk profile and outcome of Middle Eastern patients undergoing percutaneous coronary intervention. Saudi Med J. 2017;38(2):149-55.

29. Heer T, Hochadel M, Schmidt K, Mehilli J, Zahn R, Kuck KH, Hamm C, Bohm M, Ertl G, Hoffmeister HM et al: Sex differences in percutaneous coronary intervention-insights from the coronary angiography and $\mathrm{PCl}$ registry of the German society of cardiology. J Am Heart Assoc 2017, 6(3).

30. Valgimigli M, Gragnano F, Branca M, Franzone A, Baber U, Jang Y, Kimura T, Hahn JY, Zhao Q, Windecker S et al: P2Y12 inhibitor monotherapy or dual antiplatelet therapy after coronary revascularisation: individual patient level meta-analysis of randomised controlled trials. BMJ 2021, 373:n1332.

31. Cesaro A, Taglialatela V, Gragnano F, Moscarella E, Fimiani F, Conte M, Barletta V, Monda E, Limongelli G, Severino S, et al. Low-dose ticagrelor in patients with high ischemic risk and previous myocardial infarction: a multicenter prospective real-world observational study. J Cardiovasc Pharmacol. 2020;76(2):173-80.

32. Liang Z, Li Y, Wang J, Wang D, Wang S, Ma L, Liu H, Yang L, Stone GW, Han Y. The safety and effectiveness of bivalirudin in female patients with acute myocardial infarction undergoing primary angioplasty: A subgroup analysis of the BRIGHT trial. Catheter Cardiovasc Interv. 2016;87(Suppl 1):608-15.

33. Hayes SN, Tweet MS, Adlam D, Kim ESH, Gulati R, Price JE, Rose CH. Spontaneous coronary artery dissection: JACC state-of-the-art review. J Am Coll Cardiol. 2020;76(8):961-84.

\section{Publisher's Note}

Springer Nature remains neutral with regard to jurisdictional claims in published maps and institutional affiliations. 